\title{
Psychotherapy and the Professional Identity of Psychiatry in the Age of Neuroscience
}

\author{
Ren Belcher ${ }^{1,2,3}$ (ID)
}

Received: 7 June 2019 / Revised: 17 August 2019 / Accepted: 20 September 2019 / Published online: 16 November 2019 (C) Academic Psychiatry 2019

Interviewing for residency positions in psychiatry, I was asked repeatedly why I wrote in my application that I felt drawn to the field because I wanted to perform psychotherapy. Responses to this interest varied considerably. On one extreme, there are a handful of departments that regard psychotherapy as the core skill in clinical psychiatry, and at these places - I can think of a few-I had little explaining to do.

On the other hand, there are a number of programs that vow to make psychotherapy as effectively optional as they can get away with. One chief resident told me in no uncertain terms that therapy didactics and supervision were "available" to residents only because the accreditation council requires them.

Then there is the most common response, which I would describe as basically encouraging but imbued with skepticism. It reminds me of the way medical schools react when an entering student declares an interest in primary care for the poor. Every medical school wants these kinds of students in their class, but you can tell that the deans, having heard this before, are aware of the odds. They know that most of them, in keeping with the majority of like-minded medical students, will eventually get pulled in another direction $[1,2]$. Rational students succumb to market forces; likewise, a psychiatry resident's desire to perform psychotherapy might be replaced by other, more practical, considerations as they develop a career. (A notable exception exists in the handful of very large cities fostering a market for private practice therapy, but this is not the norm nationwide.)

Before moving further, I should clarify that by psychotherapy I mean all of the deliberate interactions that might be thought of as "talking cures," which take as axiom that we can bring about change in a patient's experience by sharing in

Ren Belcher

lrbelcher@partners.org

\footnotetext{
Massachusetts General Hospital, Boston, MA, USA

McLean Hospital, Belmont, MA, USA

Harvard Medical School, Boston, MA, USA
}

it. Psychotherapy is also a scientific discipline, based on measured observation, which has demonstrated beyond ambiguity that a therapist's choice to sit with a patient, to utter (or not), and to listen with purpose [3] has therapeutic action [4]. In the modern landscape, psychotherapy runs the gamut from brief to prolonged, tightly framed to spontaneous, individual to collective, suggestive to analytic, and so on, but for my argument here (and perhaps for the benefit of patients [5]), these differences are not as important as their commonalities.

By this definition, I do not think anyone disputes that many patients need psychotherapy. A common line from program directors is "we teach therapy because therapy works," enumerating (and justifying) it as one of many tools in the therapeutic shed. They point out that beyond the role of psychotherapy in the conditions we see most commonly, several epidemiologically significant psychiatric disorders have no FDA-approved medication or somatic treatment but a wealth of evidence for psychotherapeutic intervention. Borderline personality disorder [6], specific phobia [7], somatoform disorders [8], and anorexia nervosa [9] come to mind.

Of course, physicians are far from the only providers in the healthcare system. Our colleagues in internal medicine know that physical activity, nutrition counseling, and tobacco intervention programs are almost certainly more effective than some of the pharmacotherapies employed for similar ends. They are nonetheless delegated to professionals less scarce in the ecosystem because they come at an opportunity cost for prescribing physicians.

Likewise, in American psychiatry, all residents learn the basics of the major branches of psychotherapy [10], but most practicing psychiatrists today do not perform therapy with their patients, even informally, and the number of psychiatrists who perform psychotherapy has declined substantially since the 1990s [11]. Some of this is because psychotherapy has the lowest professional barrier of the modes of intervention used in psychiatry. A medical doctor can offer as much talk therapy as their heart desires, but so can a clinical psychologist, a nurse practitioner, a psychiatric nurse, a social worker, a mental health counselor - and arguably also a minister, a vocational 
counselor, and even a parole officer, "often without recognizing it as such" [12].

Our healthcare system, coding therapy as a consumable transaction just like any other procedure or office visit, gives the impression that physician-led therapy must be very expensive. In the time it takes to conduct a single therapeutic session (itself a fraction of even the most efficient time-limited therapy for a single patient), a prescriber could initiate or titrate the medications of four others. And outcomes from psychotherapy appear to be equal whether the provider is a psychiatrist, psychologist, or social worker [13].

There is actually some data to suggest that in the long run, the assumed premium of reimbursing MDs for psychotherapy does not pan out: having a doctor provide combined pharmacotherapy and psychotherapy for patients who need both is no costlier to the system than a "split treatment" with a doctor and another clinician [14]. But practice trends and insurance reimbursements do not account for this parity, perhaps because the culture of medicine does not want it. Physicians across the medical specialties rally that doctors should practice "at the top of our license" - in other words, we should spend most of our time doing tasks that no one else in the healthcare system is authorized to do. For psychiatry, this might mean prescribing clozapine or ordering brain scans or performing ECT—not conducting psychotherapy.

This stands on an implicit assumption that any given patient's psychotherapy can be outsourced without some consequence to the psychiatrist's therapeutic ability. So consider the alternative: a professional culture where psychiatrists only concern themselves with data pertaining to medical intervention, with milligrams and joules, and the latest clinical trial outcomes. What would be missed?

Perhaps what is at stake here is some professional disagreement surrounding what it is, exactly, psychiatrists do for a living. Are we brain doctors? I have a few colleagues who introduce themselves to patients as such. But there is something very satisfying to me about the argument from Josef Parnas and Pierre Bovet that the brain must not necessarily be our focus of attention:

The object of psychiatry [is not the brain but is] the patient's altered experiences, expression, and existence, associated with 'suffering in self and/or others'...The brain enters [our profession] in so far that it contributes to this suffering in a medically relevant way and not because the brain per se or de jure is of primary interest in psychiatry [15].

Clearly the brain is central to psychiatry, and our better understanding of the neurobiology influencing our patients has, and will continue to, revolutionize our work. What matters is the emphasis. Parnas and Bovet question neuroscientific exceptionalism (i.e., neuroscience is the irreducible basis of psychiatry) when faced with the "'growing disconnect' between 'the brilliant progress' of neurosciences and its 'nearly complete failure' to translate into diagnostic or therapeutic gains in psychiatry" [15]. This is made all the more salient by the ongoing prioritization of reductionist approaches to neuroscience in research funding: just one of the seven units of analysis in the NIMH's Research Domain Criteria - the unit called 'self-reports' - makes scientific room for the patient's experience [16].

Eric Turkheimer, a conceptually invested geneticist, describes these failures of translation as the joint of psychiatry's central conflict:

We can see [in this problem] both horns of the dilemma that has plagued psychiatry from the start: indulge a high-level, humanistic model of causation and explanation, paying a price in methodological rigor, or indulge an exclusively reductionist view grounded in the natural sciences, paying a price in psychological relevance [17].

Psychiatry's added value is that we are armed with both, and privileging biology over psychology is missing the point. Our task, then, is to "see more" [18] in any given psychiatric patient: to grasp, "within a single look" [18], "the one in the many and the many in the one" [19]. It is to come to a reasonable hypothesis about which levels of the mind-brain system are most germane to the problem at hand, and, in doing so, to estimate the treatments best suited for them. This role is rooted in an interdisciplinary professional training, designed to cultivate some appreciation for each of the currently discernible layers of the mind-brain system, but it also encompasses a humble understanding that the scientific narratives in each of these individual disciplines are "unable, because unsuited" [20] to capture the wholeness of the patient's presentation.

So where does this leave psychotherapy? For all we have discussed about levels in the complex mind-brain system, clearly the level requiring our greatest professional conviction is the level that patients present to us. This is alternately called behavior, chief complaint, experience, or phenotype - whatever term we use, it is the absolute essence of what we are treating. When a medical student chooses to care for mental disorders instead of cancer or lupus or pneumonia, it is with the understanding that the mind cannot be impartially observed. Brain scans tell us what they will, but suffering and illness are determined by communication. The "history" is hardly a history at all; it is affected by errors in recall, cognitive distortions, conscious and unconscious impressions the patient wishes to make on the doctor, and numerous other influences [12].

Psychotherapy, unlike any other intervention in psychiatry, speaks the language of the patient rather than the language of the brain. It interacts in real time with the experience of suffering that brings a patient to our care. And to our own benefit, by participating [21] in the patient's "experience, expression, 
and existence," we gain a more fluent and dynamic sense of its malleability - its suitedness to each of the therapeutic tools at our disposal, no matter which level they reach.

We come to this: psychotherapy is the sine qua non of psychiatry. It is not the totality of what we have to offer, its fundamentality does not devalue concurrent progress in somatic therapies, and tightly framed psychotherapy is unlikely to consume most of our effort as a profession. But psychotherapy is the discipline which establishes our manner of relating to patients, our "way in" to their experience, and our understanding of ourselves as an instrument of measurement and change. Learning psychotherapy is to learn listening with purpose [3], which is the skill on which our entire work depends.

Over the past century, in the wake of advances changing our understanding of the nature of all medical disease, psychiatry has increasingly organized its diagnostic and therapeutic paradigms around issues of legitimacy and medical validity. But even in the "golden age of brain science" [22, 23], knowing what we do about the fantastic heritability of some of our illnesses, their predictable response to neurotransmitter modulation, and the utility of a handful of emerging biomarkers, I suggest that our medical colleagues want something more from us. To revisit Turkheimer's central dilemma- - "indulge a high-level, humanistic model of causation and explanation, paying a price in methodological rigor, or indulge an exclusively reductionist view grounded in the natural sciences, paying a price in psychological relevance" [17] —I think it is clear which side of the horn better serves the medical profession.

Any clinician can count symptoms [15], diagnose an illness following consensus criteria, and offer a preliminary treatment plan for most psychiatric disorders. Indeed, more mental disorders are treated by non-psychiatrists than by psychiatrists [24]. I am training in psychiatry because I believe psychiatrists offer a different, more nuanced, and more empirical kind of care. Psychiatrists understand that subtleties truly matter in hearing a patient's story, and they know that these are inadequately captured by the diagnostic criteria other physicians rely on in our stead. Our colleagues and our patients want us to see more: to infer meaning from a patient's posture and gaze, his turn of phrase, and his manner of relating - and to offer a vision for how these may come to change. Not all psychotherapists are psychiatrists, but indeed all psychiatrists are psychotherapists. The patient is telling us more than can be counted, and other doctors trust that psychiatrists know how to listen.

Acknowledgments I am deeply grateful to Dr. Michael Marcangelo for supporting my interest in writing this paper and for his endless advice and encouragement throughout medical school. I am also thankful for the support and guidance of Dr. Deborah Spitz, who introduced me to the fascinating intellectual history of our profession. Finally, I am fortunate to have the mentorship of Dr. Catherine Kimble, who has nurtured my growing interest in psychotherapy and provided valuable comments on this article.

\section{Compliance with Ethical Standards}

Disclosures The author states that there is no conflict of interest.

\section{References}

1. Colwill JM. Where have all the primary care applicants gone? N Engl J Med. 1992;326:387-93.

2. The complexities of physician supply and demand: projections from 2014 to 2025, 2016 update. Association of American Medical Colleges; 2016. Available from: https://www.aamc.org/ download/458082/data/2016_complexities_of_supply_and_ demand projections.pdf

3. Danielian J, Gianotti P. Listening with purpose: entry points into shame and narcissistic vulnerability. Lanham: Jason Aronson, Inc.; 2012.

4. Campbell LF, Norcross JC, Vasquez MJT, Kaslow NJ. Recognition of psychotherapy effectiveness: the APA resolution. Psychotherapy. 2013;50:98-101.

5. Wampold BE, Imel ZE. The great psychotherapy debate: the evidence for what makes psychotherapy work. 2nd ed: Abingdon, United Kingdom: Routledge; 2015.

6. Gunderson JG, Herpertz SC, Skodol AE, Torgersen S, Zanarini MC. Borderline personality disorder. Nat Rev Dis Primer. 2018;4: 18029.

7. Grös DF, Antony MM. The assessment and treatment of specific phobias: a review. Curr Psychiatry Rep. 2006;8:298-303.

8. Kroenke K. Efficacy of treatment for somatoform disorders: a review of randomized controlled trials. Psychosom Med. 2007;69: 881-8.

9. Treasure J, Zipfel S, Micali N, Wade T, Stice E, Claudino A, et al. Anorexia nervosa. Nat Rev Dis Primer. 2015;1:15074.

10. ACGME Program requirements for graduate medical education in psychiatry. 2019. Available from: https://www.acgme.org/Portals/0/ PFAssets/ProgramRequirements/400_Psychiatry_2019.pdf?ver= 2019-06-19-091051-927

11. Mojtabai R, Olfson M. National trends in psychotherapy by officebased psychiatrists. Arch Gen Psychiatry. 2008;65:962-70.

12. Frank JD, Frank JB. Persuasion and healing [Internet]. 3rd ed. Baltimore, MD: Johns Hopkins University Press; 2014. p. 15.

13. Seligman ME. The effectiveness of psychotherapy. The Consumer Reports study. Am Psychol. 1995;50:965-74.

14. Dewan M. Are psychiatrists cost-effective? An analysis of integrated versus split treatment. Am J Psychiatry. 1999;156:324-6.

15. Parnas J, Bovet P. Psychiatry made easy: operation(al)ism and some of its consequences. In: Parnas KKJ, editor. Philos Issues Psychiatry III Nat Sources Hist Change, vol. 201. Oxford: Oxford University Press; 2014. p. 203.

16. Cuthbert BN, Insel TR. Toward the future of psychiatric diagnosis: the seven pillars of RDoC. BMC Med. 2013;11:126.

17. Turkheimer E. The nature of nature. Philos Issues Psychiatry III Nat Sources Hist Change. Oxford: Oxford University Press; 2014. p. 242.

18. Abbott A. The Aims of Education. Chicago: University of Chicago Record 37; 2002. Available from: https://college.uchicago.edu/ student-life/aims-education-address-2002-andrew-abbott 
19. Kendler KS. David Skae and his nineteenth century etiologic psychiatric diagnostic system: looking forward by looking back. Mol Psychiatry. 2017;22:802-807. Quotation from page 805.

20. Nordgaard J, Parnas J. A haunting that never stops: psychiatry's problem of description. Acta Psychiatr Scand. 2013;127:434-5 Quotation from page 434.

21. Basch MF. Doing psychotherapy. New York: Basic Books; 1980.

22. Neuroscience: New angles on the brain. Nature. 2013;503:21.
23. Choi OS. What neuroscience can and cannot answer. J Am Acad Psychiatry Law Online. 2017;45:278-85.

24. Regier DA, Goldberg ID, Taube CA. The de facto US mental health services system: a public health perspective. Arch Gen Psychiatry. 1978;35:685-93.

Publisher's Note Springer Nature remains neutral with regard to jurisdictional claims in published maps and institutional affiliations. 